\title{
AGE AND GENDER WISE DISTRIBUTIONOF RISK FACTORS AMONG PATIENTS WITHCORONORY ARTERY DISEASE.
}

\author{
MUHAMMAD ASIF IQBAL ${ }^{1}$, IKRAMULLAH ${ }^{1}$, ABDUL HADI ${ }^{1}$, FAROOQ AHMAD ${ }^{2}$, \\ MUHAMMAD REHANUL HAQ ${ }^{1}$, MUHAMMAD ADIL ${ }^{1}$, MUHAMMAD NAUMAN ${ }^{1}$, \\ MUHAMMAD HAFIZULLAH' \\ 1. Cardiology Department, PGMI, Lady Reading Hospital, Peshawar \\ 2. Medical Officer, Emergency Satellite Hospital, Nahaqai, Peshawar.
}

\begin{abstract}
OBJECTIVE: To describe conventional risk factors by age and gender in coronary artery disease patients.

MATERIALS AND METHODS: This study was conducted in outdoor patient department of agency headquarter hospital (AHQ) Landikotal, Khyber agency, from June to October 2013. It was a hospital based descriptive observational study. Sample size was 926 patients with established coronary artery disease, recruited through purposive convenient sampling technique. Adult patients (above 18 years) of both genders with coronary artery disease were included in the study. Every patient was evaluated for the presence of conventional risk factors. Card vascular risk factors were assessed by a self-administered questionnaire and various laboratory tests.
\end{abstract}

RESULTS: Total patients were 926, male were 546(59\%). Mean age was 58.28 \pm 12.005 years (20-95 years). Study population was divided into younger (age $\leq 40$ years) and older age groups ( $\geq 40$ years). Younger patients were 66(7.1\%) and older age 866(92.9\%). Hypertension was present in 563 patients $(61.1 \%)$ with $59.2 \%$ and $63.2 \%$ in male and female, respectively $(\mathrm{p}=0.186)$. Diabetes was present in $29.9 \%$ with $27.8 \%$ in male and $32.9 \%$ in female patients $(\mathrm{p}=0.098)$.Hypertension was significantly more common in older patients while smoking and heavy fat intake was significantly more common in younger patients. Other risk factors for CAD were similar across younger and older age groups.

CONCLUSION: Conventional risk factor in male and female differ widely with smoking, ex-smoker, heavy fat intake and obesity is significantly more common in male patients, while family history for CAD is common in female patients. Diabetes is higher in 40 years or above patients while smoking is higher in patients less than 40 years.

KEY WORDS: Conventional Risk Factor, Coronary Artery Disease, Age, Gender

\section{INTRODUCTION}

Cardiovascular diseases (CVD) are the most common cause of death in the world. According to a report of World Health Organization, about 7.6 million people died due to myocardial infarction in $2005^{1}$. Cardiovascular mortality in developed countries is decreasing, while it is increasing in developing countries. About $80 \%$ of the CVD mortality is now occurring in these countries $^{2}$.Prevalence of coronary artery disease (CAD) varies according to the study population. Its prevalence is, $7.3 \%$ in general population, $3.8 \%$ in symptomatic population and $17.1 \%$ in asymptomatic patients ${ }^{3}$.In a study, among patients with $\mathrm{CAD}$, hypertension was present in
$47.7 \%$ of patients, diabetes in $12.9 \%$, dyslipidemia in $90.1 \%$, and smoking in $24.1 \%$ of patients. Two or more risk factors were present in $66.5 \%$ of patients ${ }^{4}$. Dodani et al in their study on general population, reported that prevalence of hypertension, hypercholesterolemia and diabetes were $38.5,10.7$ and 9.1 per cent, respectively. Obesity or overweight was present in $52 \%$, 64\% were leading sedentary life and $12 \%$ had two or more risk factors for $\mathrm{CAD}^{5}$.

Hypertension, diabetes, hyperlipidemia and cigarette smoking act as independent risk factors for $\mathrm{CAD}$ in more than $50 \%$ of patients. Risk factors for $\mathrm{CAD}$ varies between men and women 
and this sex ratio varies between populations $s^{6,7,8,9}$. CAD is 2 to 5 times more common in men than in women among middleaged people. In both sexes, the risk of CAD increases markedly with age. Similarly, risk factors for $\mathrm{CAD}$ varies according to the age of presentation $^{10,11}$. The role of major cardiovascular risk factors in the development of $\mathrm{CAD}$ is well established among men. However, among women, the data is less extensive. Reasons for the sex difference in CAD risk are not fully understood ${ }^{12}$. In the Framingham study, substantial differences were noted in the risk factors for CAD that occurred before the age of 65 years. Differences were noted between men and women and among different age groups. These patterns have biological, clinical and statistical significance $^{13}$.

It is important to recognize sex differences in risk factors for CAD from public health and clinical point of view. However, very limited national data is available to assess the extent to which cardiovascular risk factors can explain the observed gender and age related differences in risk factors for $\mathrm{CAD}$ in our population.

This study was therefore, conducted to assess, whether conventional risk factors (hypertension, diabetes, hypercholesterolemia, history of premature CAD in the family, obesity, smoking, ex-smoker, heavy fat intake and sedentary life style) are similar in men and women; and to assess the difference in CAD risk between younger and older age groups in. This aim of the study was, to compare conventional risk factors for CAD between men and women and between young and older age groups in our population.

\section{MATERIALS AND METHODS}

Hospital ethical committee approved study protocol prior to start of the study. Written informed consent was taken from all study participants. This study was conducted in outpatient department of Agency Headquarter (AHQ) hospital Landikotal from June to October 2013. It was a hospital based descriptive observational study. Sample size was 926 patients. It was calculated on the basis of $7 \%$ prevalence of established $\mathrm{CAD}^{2}$, total population of the area is 100,000 with $95 \%$ confidence interval and 3 margin of error. Sampling technique was purposive convenient. Sample selection was simple random. Patients who presented to outpatient department of AHQ hospital Landikotal with CAD were evaluated for enrolment in the study. Adult patients (above18 years) of both genders who were diagnosed as CAD were included in the study. Patients with concomitant valvular heart disease and congenital heart disease were excluded from the study as these diseases make diagnosis doubtful. Patients with incomplete history or investigations were also excluded from the study.

Study population was divided into younger age (less than 40 years) and older age group (40 years and above). Every patient was evaluated for the presence of conventional risk factors. Cardiovascular risk factors were assessed by a self-administered questionnaire and various laboratory tests. Previous record of the patient was reviewed in detail for any previous procedure such as coronary angiography, percutaneous coronary intervention or previous bypass surgery. Detailed history was taken. History of daily activities, use of fats per week was sought. Similarly, history of hypertension, diabetes and past history of myocardial infarction, percutaneous coronary intervention (PCI) or coronary artery bypass graft (CABG) was determined. Previous record of the patient was reviewed in detail for any previous procedure such as coronary angiography, PCI or CABG. Present or past habit of smoking was asked. Family history of premature CAD in the family was determined. Detailed clinical examination was carried out. Blood pressure was measured with the subject in sitting position after at least five minutes' rest using mercury blood pressure apparatus. Newly diagnosed hypertension was confirmed by reevaluation after a week. Weight and height measured and Body Mass Index (BMI) calculated. BMI was calculated as weight $(\mathrm{kg})$ divided by height 
squared $\left(\mathrm{m}^{2}\right)$. Relevant investigations were performed. Fasting or random blood glucose and serum cholesterol measured, from hospital laboratory using microlab-III machine. All details were entered on a pre- determined proforma.

CAD was defined as documented CAD on coronary angiography or history of myocardial infarction or patients on anti-angina therapy. Myocardial infarction was defined as previous hospitalization for myocardial infarction as evident from previous record or new ST elevation in any two leads with ongoing chest pain on presentation. PCI: PCI was defined as previous procedure of angioplasty with placement of one or more stents as evident from record. CABG: any patient who has undergone CABG previously as evident from record. Angina: angina was defined as exertion chest pain that relieves with rest or sublingual nitroglycerin or patients already taking anti angina treatment.

Risk factors were defined as follow: Diabetes: self-reported history of diabetes or use of antidiabetic medications or fasting blood sugar $126 \mathrm{mg} \%$ or random blood sugar more than $180 \mathrm{mg} \%$. Hypertension: self-reported history of hypertension or the use of anti-hypertensive drugs or documented blood pressure more than $140 / 80 \mathrm{~mm} \mathrm{Hg}$ on more than one occasion. Hypercholesterolemia: random blood cholesterol equal or more than $200 \mathrm{mg} / \mathrm{dl}$. Family history of CAD: self-reported history of CAD in first degree relatives. Smoking: regular use of more than five cigarettes per day for more than one year. Ex-smoking: smokers who smoked for at least 2 years and quit smoking at least 6 months ago.

Obesity: BMI more than 29.9. Heavy fat intake: use of fatty meal more than 6 times a week as assessed on self-administered questionnaire.

Sedentary life style: No exertional activity and not more than 20 minutes walk daily as assessed on self-administered questionnaire.
Statistical Analysis: Data entered and analyzed using SPSS version16. Qualitative variables were presented in percentages. Quantitative variables such as age were presented in mean \pm standard variation.

\section{RESULTS}

Study population was 926 patients. Out of these male were $546(59.0 \%)$. Mean age of the study population was $58.2 \pm 12.005$ years and age ranged from 20 to 95 years. Study population was divided into younger (below 40 years) and older (40 years or above) age groups. Younger age group was $66(7.1 \%)$ and older age 866(92.9\%).

CAD patients included patients with angina, previous myocardial infarction, post PCI or CABG. Angina was the predominant presentation and was present in 848 (91.9\%) patients. Myocardial infarction was present in $379(40.9 \%)$, PCI in $63(6.8 \%)$ and CAB Gin 49 $(5.3 \%)$ patients.

Hypertension was the most common risk factor, present in $563(61.1 \%)$ patients. Hypertension in males and female was $59.2 \%$ and $63.2 \%$, respectively $(\mathrm{p}=0.186)$. Diabetes was present in $29.9 \%$ with $27.8 \%$ (male) and $32.9 \%$ (female) frequency $(\mathrm{p}=0.098 \%)$. Family history was present in $375(40.5 \%$ ) patient with 36.45 in male and $46.3 \%$ in female ( $\mathrm{p}=0.005)$.Hypertension, hypercholesterolemia and diabetes were similar in men and women. Smoking and sedentary life was significantly more common in female patients. With the exception of family history of CAD, men had more disadvantageous cardiovascular risk profile. Gender wise distribution of risk factor is given in Table-1.

Table 1: Comparison of risk factors among men and women

\begin{tabular}{|l|l|l|l|}
\hline Variable & $\begin{array}{l}\text { Men } \\
\text { Frequency } \\
(\%)\end{array}$ & $\begin{array}{l}\text { Women } \\
\text { Frequency } \\
(\%)\end{array}$ & P-VALUE \\
\hline Hypertension & $323(59.2)$ & $240(63.2)$ & 0.186 \\
\hline Diabetes & $152(27.8)$ & $125(32.9)$ & 0.098 \\
\hline Dyslipidemia & $76(13.9)$ & $45(11.8)$ & 0.356 \\
\hline Family History & $199(36.4)$ & $176(46.3)$ & 0.005 \\
\hline Smokers & $77(14.1)$ & $3(0.8)$ & 0.000 \\
\hline Ex-smoker & $268(49.1)$ & $9(2.4)$ & 0.000 \\
\hline
\end{tabular}




\begin{tabular}{|l|l|l|l|}
\hline obesity & $156(28.6)$ & $79(20.8)$ & 0.007 \\
\hline Sedentary life & $332(60.8)$ & $66(17.4)$ & 0.000 \\
\hline Heavy fat intake & $328(60.1)$ & $92(24.2)$ & 0.000 \\
\hline
\end{tabular}

Male were having more numbers of risk factor as compared to female patients. Among male,50\% patients had more than three risk factor while only $23 \%$ female patients had more than three risk factors $(\mathrm{p}<0.0001)$.

According to age groups analysis, hypertension was significant more common in older patients while smoking and use of heavy fat diet was significantly more common in younger patients. Other risk factors for CAD were similar across younger and older patients. (Table.2)

Table 2: Comparison of risk factors in younger and older age groups

\begin{tabular}{|l|l|l|l|}
\hline Variable & $\begin{array}{l}\text { Above 40 } \\
\text { years (\%) }\end{array}$ & $\begin{array}{l}\text { Below 40 } \\
\text { years (\%) }\end{array}$ & P Value \\
\hline Hypertension & $61.5(533)$ & $50.0(30)$ & 0.175 \\
\hline Diabetes & $30.9(268)$ & $13.3(8)$ & 0.001 \\
\hline Dyslipidemia & $12.6(109)$ & $20.0(12)$ & 0.099 \\
\hline Family History & $40.5(351)$ & $40.0(24)$ & 0.896 \\
\hline Smokers & $8.2(71)$ & $28.3(17)$ & 0.001 \\
\hline Ex-smoker & $30.9(268)$ & $20.0(12)$ & 0.075 \\
\hline obesity & $24.7(214)$ & $35.0(21)$ & 0.077 \\
\hline Sedentary life & $41.5(358)$ & $33.0(20)$ & 0.182 \\
\hline Heavy fat intake & $44.7(387)$ & $55.0(33)$ & 0.121 \\
\hline
\end{tabular}

\section{DISCUSSION}

In this study, significant differences were noted in the conventional risk factors among male and female. Smoking, heavy fat intake and sedentary life style were significantly more common in male patients, while family history of CAD was significantly more common in female patient. Our findings are consistent with international studies with few exceptions.

One study from India $^{14}$ observed that hypertension $(36.4 \%$ males. $37.5 \%$ females), diabetes (males 13.1, females $11.3 \%$ ), obesity (male $57.4 \%$, female $68.4 \%$ ) and physical inactivity (male $28.5 \%$, female $22.7 \%$ ) were similar among men and women. However, significant difference was noted in smoking (36.5\% male, $11.7 \%$ female $(\mathrm{p}<0.0001)$ and hypercholesterolemia (male $37.4 \%$, female $4.1 \%$ $\mathrm{p}<0.001$ ). Overall, CAD frequency was $6.18 \%$ and $10.12 \%$ in male and female respectively.
They concluded that there is high prevalence of hypertension, truncal obesity, diabetes and dyslipidemia and increased significantly in both males and females. It is clear from this and our study that south Asian patients have similar characteristics with regard to risk factors for CAD.

Inherent study ${ }^{15}$ found that smoking ,raised ApoB/ApoA1, history of hypertension, diabetes, abdominal obesity, psychological factors, daily consumptions of fruits and vegetables, regular alcohol consumption, and regular physical activity were all significantly related to myocardial infarction in both men and women, old and young, and in all regions of the world( $p<0.0001$ for all risk factors). Collectively, these nine risk factors accounted for $90 \%$ of the Population Attributable Risk in men and 94\% in women. In another study, males and females presented at similar ages and similar prevalence of smoking, diabetes, hypertension and family history of premature CAD, albeit women were less likely than man to have a prior history of myocardial infarction. The observed age difference between females and males with CAD was less than expected $(3.3 \pm 2.3$ years, $95 \%$ confidence limits ${ }^{16}$ ).

In a study from Palastine ${ }^{17}$, smoking was present in $60.8 \%$ males while no single female was smoker ( $\mathrm{p}$-value<0.001). However, hypertension and diabetes showed high prevalence in female than male with significant level for hypertension $(p=0.041)$. Dyslipidemia and family history of CAD showed no greater difference in both genders ${ }^{17}$. In our patients, hypertension, diabetes and dyslipidemia were equivalent in male and female. There was a trend towards high prevalence of hypertension in female patients that was statistically not significant. Hypertension was present in more than half patients and diabetes in more than one third patients. We have high frequency of hypertension, diabetes, sedentary life style and heavy fat intake. However, smoking is less prevalent in this area especially in female population. It is because, in our cultural and 
custom values, smoking especially, in women is considered unacceptable.

In our study, diabetes was significantly more common in elderly patients while smoking was significantly higher in younger patients. Jameelet $\mathrm{al}^{17}$ found that difference in risk factors for young and older age group was significant. Older patients in both gender expressed higher prevalence of hypertension, diabetes and dyslipidemia. In both age groups, females expressed high prevalence of hypertension and diabetes compared to male, but did not reach statistical significant level. Dyslipidemia was higher among male regardless of age difference. In western study, among patient with CAD, $80.6 \%$ men and $84.6 \%$ of women were having at least 1 of the 4 conventional risk factors(cigarette smoking, diabetes, hyperlipidemia and hypertension) while, only $10 \%$ to $15 \%$ of patients, in younger patients (men $\leq 55$ years and women $\leq 65$ years) were lacking any of the 4 conventional risk factors. Premature CAD was related to cigarette smoking in men and cigarette smoking and diabetes in women. Smoking decreased the age at the time of CAD event nearly a decade in all risk factors combinations ${ }^{18}$. From these studies, it is clear that CAD risk factors vary significantly among men and women and at different ges. Its distribution varies in different parts of the world. In our population, Hypertension and diabetes is higher in older and smoking is high in male patients and in younger age. We need to improve the diagnosis, treatment and compliance for hypertension and diabetes. Similarly, smoking is easily controllable risk factor. Addressing these modifiable risk factors at clinical and public health level can effectively reduce the burden of CAD in our society.

\section{CONCLUSION}

Conventional risk factors in male and female differ widely with smoking, ex smoking, heavy fat intake and obesity is significantly more common in male patients while family history for $\mathrm{CAD}$ is common in female patients. Frequency of diabetes and hypertension increases as the age advances while smoking is higher in younger age group and its frequency decreases as age advances.

\section{REFERENCES}

1. Christopher C. Anyadubalu. The experiences of coronary artery disease: biopsychosocial perspectives. International journal of human and Social Sciences2010;5:10.World Health Organization, "Report on cardiovascular disease 2005".

2. Yusuf S, Reddy S, Ôunpuu S, Anand S. Global burden of cardiovascular diseases, part I: general considerations, the epidemiologic transition, risk factors, and impact ofurbanization.Circulation 2001;104:2746-53

3. Enbergs A, Bürger R, Reinecke H, Borggrefe M, Breithardt G, KerberS.Prevalence of coronary artery disease in a general population without suspicion of coronary artery disease: angiographic analysis of subjects aged 40 to 70 years referred for catheter ablation therapy. Eur Heart J2000;21(1):45-52.

4. Rinkūniene E, Petrulioniene Z, Laucevicius A, Ringailaite E, Laucyte A. Prevalence of conventional risk factors in patients with coronary heart disease. Medicina (Kaunas). 2009;45(2):140-6.

5. Dodani S, Mistry R, Khwaja A, Farooqi M, Qureshi R, Kazmi K. Prevalence and awareness of risk factors and behaviours of coronary heart disease in an urban population of Karachi, the largest city of Pakistan: a community survey. J Public Health 2004;26:(3):245-9.

6. Castelli WP. Epidemiology of coronary heart disease: the Framingham Study. Am J Med 1984;76:4-12.

7. Thelle D. Women and coronary heart disease: a review with special emphasis on some risk factors. Lipid Rev1990;4:33-9.

8. Thom TJ, Epstein FH, Feldman JJ, Leaverton PE, Wolz M. Total Mortality and Mortality From Heart Disease, Cancer and Stroke From 1950 to 1987 in 27 Countries. Bethesda, Md: National Institutes of Health. NIH publication1992;3088-92.

9. Kuhn FE, Rackley CE. Coronary artery disease in women: risk factors, evaluation, treatment, and prevention. Arch Intern Med1993;153:2626-36.

10. Tunstall-Pedoe H, Kuulasmaa K, Amouyel P, Arveiler D, Rajakangas A-M, Pajak A). Myocardial infarction and coronary deaths in the World Health Organization MONICA Project: registration procedures, event rates, and case-fatality rates in 38 populations from 21 countries in four continents. Circulation1994;90:583-612.

11. Rich-Edwards JW, Manson JAE, Hennekens CH, Buring JE. The primary prevention of coronary heart disease in women. $N$ Engl J Med1995;332:1758-66. 
12. Jackson R, Chambless L, Higgins M, Kuulasmaa K, Wijnberg L, Williams D (WHO MONICA Project, and ARIC Study). Sex difference in ischaemic heart disease mortality and risk factors in $\mathbf{4 6}$ communities: an ecologic analysis.Cardiovasc Risk Factors 1997;7:43-54.

13. Stokes J 3rd, Kannel WB, Wolf PA, Cupples LA, D'Agostino RB.The relative importance of selected risk factors for various manifestations of cardiovascular disease among men and women from 35 to 64 years old: 30 years of follow-up in the Framingham Study. Circulation 1987;75:65-73.

14. GuptaR,GuptaVP,SarnaM,BhatnagarS,ThanviJ,Shar maV,Singh AK, etal.Prevalence of coronary artery disease and risk factors in an urban Indian population:Jaipur Heart Watch-2.Indian Heart J2002;54(1):59-66.

15. YusafS,Hawken S, Ounpuu S, Dans D, Avezum A, LanasF,McQueenM,BudajA,PaisP,Varigos

J,(INTERHEART Investigators).Effect of potentially modifiable risk factors associated with myocardial infarction in 52 countries(the INTERHEART study):case control study. The Lancet 2004;364:937952.

16. Jong P,MohammadS,Sternberg L. Sex differences in the features of coronary artery disease of patients undergoing coronary angiography. Can J Cardiol1996;12(7):671-7

17. JameeA,AbedY,Jalambo OM. Gender difference and characteristics attributed to coronary artery disease in Gaza Palestine. Global Journal of Health Sciences 2013;5:50-6.

18. KhotUN.KhotBM,BajzerCT,SappSK,Ohman M, BrenerSJ,Ellis SJ et al.Prevalence of conventional risk factors in patients with coronary heart disease.JAMA2003;290(7):898-904.

CORRESPONDENCE ADDRESS:

Dr. Muhammad Asif Iqbal

Cardiology Department,

Lady Reading Hospital, Peshawar, Pakistan

Email: drasifiqbal_175@yahoo.com

Cell no: 00-92-333-918-7404 